\title{
DEPRESSÃO PÓS-AVC: FATORES DE RISCO E TERAPÊUTICA ANTIDEPRESSIVA
}

\author{
Luisa de Marillac Niro Terroni, Claudia Costa Leite, Gisela tinone, Renério fráguas JR* \\ Trabalho realizado no Instituto e Departamento de Psiquiatria, Departamento de Radiologia - \\ Setor de Ressonância Magnética e Divisão de Clínica Neurológica da \\ Faculdade de Medicina da Universidade de São Paulo, São Paulo, SP.
}

RESUMO - A depressão é a complicação psiquiátrica mais freqüente nos pacientes com acidente vascular cerebral (AVC). Vários aspectos têm sido detectados como fatores de risco para a sua ocorrência. Neste artigo faz-se uma revisão dos fatores envolvidos na depressão pós-AVC e o estado atual de seu tratamento, a fim de estimular sua detecção e adequado tratamento pelo médico nãopsiquiatra.

A prevalência da depressão maior pós-AVC é de $10 \%$ a $34 \%$, variando conforme as diferenças dos métodos de pesquisa. 0 período do pós-AVC, o tipo de população avaliada e o tratamento recebido pelos pacientes, assim como o critério utilizado para 0 diagnóstico da depressão, podem influir a sua prevalência.

Fatores de risco associados à ocorrência da depressão pós-AVC têm sido detectados, tais como: prejuízo funcional, prejuízo cognitivo, história de depressão no passado, idade, sexo, AVC prévio, hipercortisolemia, precária rede de suporte social e carac- terísticas neuroanatômicas do AVC. Estes têm fornecido suporte para formulação de um mecanismo fisiopatológico da depressão pós-AVC, relacionado às vias prefrontosubcortical e à neurotransmissão das aminas biogênicas.

As repercussões da depressão são significativas, incorrendo em um maior grau de prejuízo funcional, retardo do processo de reabilitação, complicações na evolução e maior risco de mortalidade. A isto se soma o seu subdiagnóstico e subtratamento.

Com 0 advento da ressonância magnética, pesquisadores devem investigar a associação de regiões cerebrais específicas com a manifestação depressiva e resposta terapêutica. Aspectos metodológicos devem ser levados em consideração para uma análise mais confiável.

UnITERMOS: Depressão. Acidente Cerebrovascular. Fatores de Risco. Tratamento.

\section{INTRODUÇÃO}

O acidente vascular cerebral (AVC) pode limitar de modo significativo o desempenho funcional, com conseqüências negativas nas relações pessoais, familiares, sociais e sobretudo na qualidade de vida. Essa limitação, entretanto, nem sempre se deve ao déficit neurológico em si. Complicações psiquiátricas têm sido indicadas como fatores determinantes da incapacitação do paciente após o AVC. Dentre as complicações psiquiátricas, a depressão é a mais prevalente e a que mais tem sido associada a um pior prognóstico.

Conhecer a natureza desta depressão e seus fatores de risco torna-se essencial para aprimorar seu diagnóstico e tratamento.

A participação de fatores psicológicos é difícil de ser questionada. Somente o impacto

\footnotetext{
* Correspondência:

Instituto de Psiquiatria do HC-FMUSP

Grupo de Interconsultas-Projeto Depressões Secundárias

Rua Ovídio de Campos s/n, $3^{\circ}$ andar - sala 4017 05403-010 - São Paulo/SP

Tel.:(1 1)3069-6585/3085-3531

rfraguas@hcnet.usp.br
}

psicológico gerado pelas limitações impostas pelo AVC pode ser o suficiente para originar um quadro depressivo com um matiz de ajustamento/reativo. Estudos iniciais, entretanto, já sugeriram que além do aspecto psicológico deveria existir uma contribuição orgânica na etiologia da depressão pós-AVC. Folstein et al.' relataram uma maior prevalência da depressão pós-AVC quando comparada com pacientes ortopédicos com limitação física semelhante. Robinson e Szetela ${ }^{2}$ encontraram uma associação da depressão pós-AVC com a localização deste na região frontal anterior esquerda. Deste modo, pelo menos alguns quadros de depressão pós-AVC devem decorrer diretamente de alterações fisiopatológicas do AVC (síndrome mental orgânica ou depressão secundária ao AVC).

Nos últimos 20 anos vários estudos vêm tentando confirmar e delimitar a participação orgânica na etiologia da depressão pós-AVC, no entanto os resultados nem sempre corroboram os dados iniciais. Embora achados negativos, como a não-detecção da associação entre a localização do AVC e a ocorrência da depres- são, têm sido utilizados, por alguns, para considerar os fatores psicológicos como a principal, se não a única etiologia do quadro depressivo pós-AVC ${ }^{3}$, vários estudos suportama participação do comprometimento neuronal pelo AVC na etiologia da depressão. Esta revisão tem como objetivo comentar os estudos até aqui realizados para investigar os fatores de risco para a depressão pós-AVC, bem como o estado atual de seu tratamento antidepressivo. Para tanto, a partir do MEDLINE foram levantados os estudos a este respeito nos últimos 10 anos (unitermos: depression x stroke). Foram incluídas publicações anteriores relevantes citadas nestes artigos.

\section{Epidemiologia}

O AVC é a terceira causa de morte no município de São Paulo, antecedido apenas pelas doenças coronarianas e homicídio ${ }^{4}$. Nos Estados Unidos são registrados 400.000 casos novos por ano $0^{5}$. Um estudo transversal oriundo do Longitudinal Aging Study Amsterdam (LASA) detectou uma prevalência de AVC de $5,7 \%$ numa população de 55 a 85 anos de idade. 


\begin{tabular}{|c|c|c|c|c|c|c|c|c|}
\hline \multicolumn{9}{|c|}{ Tabela I - Prevalência da depressão pós-AVC } \\
\hline Estudo & População & $\mathbf{N}$ & $\begin{array}{l}\text { Instrumento } \\
\text { diagnóstico }\end{array}$ & Tempopós-AVC & $\begin{array}{l}\text { Depressão } \\
\text { maior }\end{array}$ & $\begin{array}{l}\text { Depressão } \\
\text { menor }\end{array}$ & $\begin{array}{l}\text { Depressão por } \\
\text { outro criterio }\end{array}$ & Total \\
\hline $\begin{array}{l}\text { Robinson } \\
\text { etal. } 1983^{9}\end{array}$ & I64H & 103 & $\begin{array}{c}\text { PSE, DSMIII } \\
\text { ZUNG, HAM-D }\end{array}$ & $2 \mathrm{sem}$ & $27 \%$ & $20 \%$ & - & $47 \%$ \\
\hline $\begin{array}{l}\text { Robinson } \\
\text { etal. } 1984^{10}\end{array}$ & $103 \mathrm{~A}$ & 61 & $\begin{array}{c}\text { PSE, DSMIII } \\
\text { ZUNG, HAM-D }\end{array}$ & $\begin{array}{l}3 \text { meses } \\
6 \text { meses }\end{array}$ & $\begin{array}{l}22 \% \\
34 \%\end{array}$ & $\begin{array}{l}27 \% \\
26 \%\end{array}$ & $\begin{array}{l}- \\
-\end{array}$ & $\begin{array}{l}49 \% \\
60 \% \\
\end{array}$ \\
\hline $\begin{array}{l}\text { Robinson } \\
\text { et al. } 1987^{\prime \prime}\end{array}$ & $103 \mathrm{~A}$ & 65 & $\begin{array}{c}\text { PSE, DSMIII } \\
\text { HAM-D,ZUNG }\end{array}$ & $\begin{array}{l}12 \text { meses } \\
24 \text { meses }\end{array}$ & $\begin{array}{l}14 \% \\
21 \%\end{array}$ & $\begin{array}{l}19 \% \\
21 \% \\
\end{array}$ & $\begin{array}{l}- \\
-\end{array}$ & $\begin{array}{l}33 \% \\
42 \% \\
\end{array}$ \\
\hline $\begin{array}{l}\text { Eastwood } \\
\text { etal. 1989'3 }\end{array}$ & I06R & 87 & $\begin{array}{c}\text { SADS; RDC, ZUNG } \\
\text { GDS, HAM-D }\end{array}$ & I-3 meses & $10 \%$ & $40 \%$ & - & $50 \%$ \\
\hline $\begin{array}{l}\text { Ngetal. } \\
1995^{14}\end{array}$ & $90 R$ & 52 & DSMIIIR & $\begin{array}{c}\text { Primeiromês } \\
\text { HAM-D }\end{array}$ & - & - & $\begin{array}{l}\text { I:dep severa } \\
\text { 10:depmod } \\
\text { 18: depleve }\end{array}$ & $55 \%$ \\
\hline $\begin{array}{l}\text { Burvilletal. } \\
1995^{15}\end{array}$ & $492 \mathrm{C}$ & 248 & PSEM, DSMIII & 4 meses & $15 \%$ & $8 \%$ & - & $23 \%$ \\
\hline $\begin{array}{l}\text { Kotila etal. } \\
1998^{12}\end{array}$ & $594 \mathrm{~A}$ & 321 & $\mathrm{BDI}$ & $\begin{array}{l}3 \text { meses } \\
12 \text { meses }\end{array}$ & $\begin{array}{l}- \\
-\end{array}$ & $\begin{array}{l}- \\
-\end{array}$ & $\begin{array}{l}41 \%-54 \% \\
42 \%-55 \%\end{array}$ & \\
\hline
\end{tabular}

$\mathrm{A}=$ população ambulatorial, $\mathrm{C}=$ população da comunidade, $\mathrm{H}=$ população hospitalar, $\mathrm{R}=$ população de centro de reabilitação, $\mathrm{BD} \mathrm{I}=$ Inventário de depressão de Beck, DSM = Manual diagnóstico e estatístico dos transtornos mentais, GDS = Escala de depressão em Geriatria,, HAM-D = Escala de depressão de Hamilton, PSE = Present State Examination, $\mathrm{RDC}=$ Research diagnostic criteria, SADS = Roteiro para distúrbios afetivos e esquizofrenia, ZUNG = Escala de auto-avaliação da depressão de Zung

De acordo com o trabalho norte-americano Nacional Comorbidity Survey - (NCS), a prevalência da depressão maior na população geral é de $17,1 \%$ ao longo da vida e $10 \%$ nos 12 meses $^{7}$. No momento da avaliação (considerando 30 dias anteriores) 0 NCS encontrou uma prevalência de $4,9 \%$. No pós-AVC a taxa é bem maior, variando de $10 \%$ a $34 \%$ no momento da avaliação-15. Esta variação de prevalência da depressão pós-AVC é influenciada por vários fatores como: o período pós-AVC no qual a avaliação foi realizada, a definição de depressão, o tipo de assistência que os pacientes receberam, bem como a origem da população estudada (Tabela I).

Os estudos de Robinson et al., $, 10,11$ ilustram a variação da prevalência da depressão pós-AVC de acordo com o período avaliado. Robinson et al., $, 10,11$ acompanharam por dois anos pacientes no pósAVC e relataram as seguintes prevalências, respectivamente de depressão maior e distimia, em função do tempo pós-AVC: a) duas semanas $27 \%$ e $20 \%$; b) três meses $22 \%$ e $27 \%$; c) seis meses $34 \%$ e $26 \%$; d) 12 meses I4\% e 19\%; e) 24 meses $21 \%$ e $21 \%$. O curso natural da depressão maior pós-AVC foi de aproximadamente um ano e, de modo geral, aqueles que estavam deprimidos no segundo ano pós-AVC não eram os mesmos com depressão no primeiro ano.

O critério usado para definir a depressão também pode contribuir para diferenças de prevalência mesmo ao se avaliar populações semelhantes. Kotila et al. ${ }^{12}$ definiram depressão utilizando o ponto de corte de 10 do Inventário de Depressão de Beck (BDI) para avaliar cerca de $32 \mathrm{I}$ sujeitos selecionados de um grupo de 594 pacientes com AVC. A prevalência de depressão no décimo segundo mês foi de $55 \%$. Já no estudo de Robinson et al. " ", que utilizaram o Present State Examination (PSE) e os critérios do Manual Diagnóstico e Estatístico dos Transtornos Mentais (DSM-III), a prevalência de depressão no mesmo período pós-AVC foi de $33 \%$.

A existência ou não de uma intervenção assistencial adequada também pode influir na prevalência da depressão. Kotila et al. ${ }^{12}$ relataram uma prevalência de depressão de $41 \%$ no terceiro mês após o AVC em pacientes submetidos a um programa de assistência especializada e uma prevalência de $54 \%$ em pacientes do grupo controle sem assistência especializada (OR 0.59). No décimo segundo mês, a prevalência de depressão foi $42 \%$ e $55 \%$ (OR 0.55), respectivamente nos grupos com e sem tratamento especializado.

\section{Fatores de risco}

Os aspectos que têm sido identificados como fatores de risco para o desenvolvimento da depressão pós-AVC são: prejuízo funcional, prejuízo cognitivo, história de depressão, idade, sexo, história de AVC prévio, hipercortisolemia, aspectos sociais e correlatos neuroanatômicos (Tabela 2). A seguir, para fins didáticos, a participação destes fatores será comentada individualmente.

\section{Prejuízo funcional}

O prejuízo funcional é caracterizado pelo grau de incapacidade para realizar determinadas atividades devido a um comprometimento neurológico. Instrumentos específicos como a escala de Barthel, avaliações de atividades de vida diária e medidas de independência têm sido utilizadas para valorar o grau de prejuízo funcional.

Em centros de reabilitação, pacientes apresentaram uma associação entre o prejuízo funcional e depressão menor e grau de sintomatologia depressiva, mas não com depressão maior, sugerindo a existência de dois tipos distintos de transtornos depressivos, onde apenas o mais leve decorreria de uma reação adaptativa ao prejuízo funcional ${ }^{16}$. No primeiro mês pós-AVC, em pacientes admitidos em um centro de reabilitação, o prejuízo 


\section{Tabela 2 - Fatores considerados de risco associados ao desenvolvimento da depressão pós-AVC}

\begin{tabular}{|c|c|c|c|c|c|}
\hline Estudo & População & Fatores de exclusão & Instrumentos & Características & Fator de risco associado à depressão pós-AVC \\
\hline $\begin{array}{l}\text { Finklestein } \\
\text { et al. } 1982^{25}\end{array}$ & 38 Havaliados & $\begin{array}{l}\text { História de doença psiquiátrica } \\
\text { e endocrinopatia }\end{array}$ & HAMD & $\begin{array}{l}\text { Caso/controle e II } \\
\text { a III dias de pós-AVC }\end{array}$ & $\begin{array}{l}\text { TSD alterado fortemente associado com o distúrbio moderado a } \\
\text { severo do humor, do apetite e do sono. Índice de recuperação } \\
\text { das alterações do humor e vegetativas pós-AVC }\end{array}$ \\
\hline $\begin{array}{l}\text { Robinson } \\
\text { et al. } 1983^{9}\end{array}$ & $\begin{array}{l}164 \mathrm{H} \\
103 \text { avaliados }\end{array}$ & $\begin{array}{l}\text { Alteração de consciência } \\
\text { Afasia }\end{array}$ & $\begin{array}{l}\text { DSMIII, PSE } \\
\text { ZUNG,HAMD }\end{array}$ & $\begin{array}{l}\text { Corte transversal } \\
2 \text { semanas após o AVC }\end{array}$ & $\begin{array}{l}\text { Idade mais jovens, funcionamento social anterior ao AVC } \\
\text { Prejuízo intelectual, prejuízo funcional }\end{array}$ \\
\hline $\begin{array}{l}\text { Robinson } \\
\text { et al. } 1984^{17}\end{array}$ & $\begin{array}{l}\text { I03H } \\
61 \text { avaliados }\end{array}$ & $\begin{array}{l}\text { Alteração de consciência } \\
\text { Afasia }\end{array}$ & $\begin{array}{l}\text { DSMIII,PSE } \\
\text { ZUNG,HAMD }\end{array}$ & $\begin{array}{l}\text { Prospectivo } 2 \text { semanas, } \\
3 \text { e } 6 \text { mesesa após o AVC }\end{array}$ & $\begin{array}{l}\text { Aumento da força da associação entre a gravidade da depressão e } \\
\text { prejuízo funcional ao longo do tempo. Funcionamento social e cognitivo }\end{array}$ \\
\hline $\begin{array}{l}\text { Lipsey } \\
\text { et al. } 1985^{26}\end{array}$ & $\begin{array}{l}65 \mathrm{R} / \mathrm{A} \text {, admissões } \\
\text { consecutivas } \\
\text { avaliadas }\end{array}$ & Déficitdecompreensão & $\begin{array}{l}\text { DSMIII, HAM-D } \\
\text { ZUNG,PSE }\end{array}$ & $\begin{array}{l}\text { Prospectivo Período } \\
\text { pós-AVC }<\text { I ano }\end{array}$ & TSD alterado associado à depressão maior \\
\hline $\begin{array}{l}\text { Morris } \\
\text { et al. } 1992^{23}\end{array}$ & $\begin{array}{l}99 R \\
88 \text { avaliados }\end{array}$ & $\begin{array}{l}\text { Depressãoatual imediatamenteanterior } \\
\text { ao AVC ou por } 2 \text { anos antes, Alt. } \\
\text { Consciência,Afasia,Ansiedadepós-AVC }\end{array}$ & $\begin{array}{l}\text { GHQ, DSMII } \\
\text { CIDI,MADRS }\end{array}$ & $\begin{array}{l}\text { Transversal (cross-sectional } \\
\text { design) } 2 \text { meses após } \\
\text { oAVC }\end{array}$ & $\begin{array}{l}\text { História pessoal de t. afetivo e ansioso (dep maior)-OR3.83, Idade: } \\
\text { mais velhos(depmaior)-OR I.I,Sexo masculino(dep menor)-OR25.4 } \\
\text { Maior incapacidadefuncional (dep menor)-OR0.68 Neuroticismo }\end{array}$ \\
\hline $\begin{array}{l}\text { Astrom } \\
\text { et al. } 1993^{29}\end{array}$ & $\begin{array}{l}98 \mathrm{H} \\
70 \text { avaliados } \\
63: 3 \text { meses } \\
61: \text { I ano } \\
45: 3 \text { anos }\end{array}$ & $\begin{array}{l}\text { Obesidade, Febre } \\
\text { Desnutrição, Tireoidopatia } \\
\text { Insuficiênciahipofisária } \\
\text { D Mellitus não controlada } \\
\text { Abstinênciaálcool edrogas } \\
\text { Epilepsia, Desorientação }\end{array}$ & DSMIII & $\begin{array}{l}\text { Prospectivo } \\
3 \text { meses, I e } 3 \text { anos } \\
\text { após oAVC }\end{array}$ & $\begin{array}{l}\text { Hipercotisolemia no } 3^{\circ} \text {. mês associada à depressão maior nos } \\
\text { primeiro e terceiro anos }\end{array}$ \\
\hline $\begin{array}{l}\text { Harneyetal. } \\
1993^{27}\end{array}$ & $\begin{array}{l}\mathrm{I} 2 \mathrm{H} \\
\text { avaliados }\end{array}$ & $\begin{array}{l}\text { Doenças neurológicas, } \\
\text { Hipertensãoarterial, } \\
\text { tratamento com } \\
\text { estrógenos, endocrinopatia, } \\
\text { diabete mellitus não controlado, } \\
\text { doençafísica grave, febre, } \\
\text { desidratação, vômito persistente. } \\
\text { Medicamentos que interferemnoTSD } \\
\text { História pessoal oufamiliar de } \\
\text { transtornoafetivo ou esquizofrenia }\end{array}$ & $\begin{array}{l}\text { DSMIII } \\
\text { HAMD }\end{array}$ & $\begin{array}{l}\text { Prospectivo I e3 } \\
\text { semanas para TSD } \\
\text { e } 3 \text { a } 4 \text { semanas } \\
\text { paraavaliaçãopsiquiátrica }\end{array}$ & $\begin{array}{l}\text { Nenhum paciente recebeu diagnóstico de depressão maior } \\
\text { TSD alterado }(>4.0 \mu \mathrm{g} / \mathrm{dl}) \text { associado a maiores escores da HAMD }\end{array}$ \\
\hline $\begin{array}{l}\text { Morrisetal. } \\
1994^{16}\end{array}$ & $\begin{array}{l}100 \mathrm{R} \\
94 \text { avaliados }\end{array}$ & $\begin{array}{l}\text { Alteraçãodeconsciência } \\
\text { Afasiasevera }\end{array}$ & $\begin{array}{l}\text { DSMIII } \\
\text { MADRS }\end{array}$ & $\begin{array}{l}\text { Prospectivo 2 e } 15 \text { meses } \\
\text { apósoAVC }\end{array}$ & $\begin{array}{l}\text { Frequêência de AVCs prévios (dep menor), Prejuízo funcional } \\
\text { (dep menor): quanto maior disfunção maior sintomatologia }\end{array}$ \\
\hline \multicolumn{6}{|l|}{$\begin{array}{l}\text { mesmaamostrade } \\
\text { Morrise etal. }{ }^{23}\end{array}$} \\
\hline $\begin{array}{l}\text { Sharpeetal. } \\
1994^{20}\end{array}$ & $\begin{array}{l}515 \mathrm{C} \\
60 \text { avaliados }\end{array}$ & $\begin{array}{l}\text { Infartos múltiplos,AVCnão } \\
\text { visualizado emTC } \\
\text { Hemorragiasubaracnóide }\end{array}$ & $\begin{array}{l}\text { DSMIII } \\
\text { RSCID }\end{array}$ & $\begin{array}{l}\text { Transversal 3a5anos } \\
\text { apósoAVC }\end{array}$ & $\begin{array}{l}\text { Prejuízo funcional, sexo feminino volume do AVC (dado avaliado no } \\
\text { estudo de } 1990 \text { também e citado adiante no correlato neuroanatômico) }\end{array}$ \\
\hline $\begin{array}{l}\text { Andersen etal. } \\
\text { 199521 }\end{array}$ & $\begin{array}{l}320 \mathrm{H} \\
259 \text { avaliados } \\
\text { I ano: } 19 \mid \mathrm{C} \\
\text { avaliados }\end{array}$ & $\begin{array}{l}\text { Hemorragiasubaracnóide } \\
\text { Binswanger,Processo } \\
\text { degenerativo/expansivo do } \\
\text { SNC, História det. mental } \\
\text { exceto depressão I ano antes, } \\
\text { Alteração de consciência, } \\
\text { Demência,Afasia }\end{array}$ & $\begin{array}{l}H A M \geq 13 \\
H A M \geq 18 \\
\text { dep maior } \\
\text { BDI }\end{array}$ & $\begin{array}{l}\text { Prospectivo } 7 \text { dias } \\
1,6,12 \text { meses } \\
\text { apósoAVC }\end{array}$ & $\begin{array}{l}\text { HistóriadeAVCprévio, sexo feminino, morando sozinho, estressesocial } \\
6 \text { meses antes do AVC, I ano: diminuição das atividades sociais apóso } \\
\text { AVC, história de depressão prévia, choro patológico no primeiro mês, } \\
\text { prejuízo cognitivo no primeiro mês (+ importante) }\end{array}$ \\
\hline $\begin{array}{l}\text { Burvill } \\
\text { et al. } 1995^{15}\end{array}$ & $\begin{array}{l}\text { 498, C 248: } \\
4^{\circ} \text {. mês, 233: } \\
12^{\circ} \text {. mêsavaliados }\end{array}$ & $\begin{array}{l}\text { Demênciasevera } \\
\text { Afasia severa }\end{array}$ & $\begin{array}{l}\text { PAS, DSMIII } \\
\text { PSE }\end{array}$ & $\begin{array}{l}\text { Prospectivo } 4 \text { e } 12 \text { meses } \\
\text { apósoAVC }\end{array}$ & $\begin{array}{l}\text { Sexomasculino } \\
\text { Vivendosozinho }\end{array}$ \\
\hline $\begin{array}{l}\text { Nget al. } \\
1995^{14}\end{array}$ & $\begin{array}{l}90 R \\
52 \text { avaliados }\end{array}$ & $\begin{array}{l}\text { Afasia,Alteraçãodeconsciência } \\
\text { Delírio, Demência }\end{array}$ & $\begin{array}{l}\text { DSMIIIR } \\
\text { HAMD }\end{array}$ & $\begin{array}{l}\text { Prospectivo 22dias } \\
\text { apósoAVC }\end{array}$ & $\begin{array}{l}\text { Históriadedepressão(reativas) } \\
\text { Sexofeminino,graudeprejuízofuncional }\end{array}$ \\
\hline $\begin{array}{l}\text { Pohyasvaara } \\
\text { et al. } 1998^{18}\end{array}$ & $\begin{array}{l}486 \text { C } \\
\text { (sérieconsecutiva) } \\
277 \text { avaliados }\end{array}$ & $\begin{array}{l}\text { Afasiasevera,Alteração } \\
\text { deconsciência }\end{array}$ & $\begin{array}{l}\text { DSMIIIR } \\
\text { PSE,BDI } \\
\text { MADRS }\end{array}$ & $\begin{array}{l}\text { Prospectivo } 3 \text { a } 4 \text { meses } \\
\text { apósoAVC }\end{array}$ & $\begin{array}{l}\text { Atividadedevidadiária-graude dependência:depressãopós-AVC(ORI.8) } \\
\text { depressãomaiorpósAVC(OR2.9),Históriadedepressão:Depressão } \\
\text { pós-AVC(OR2.3), depressão maior pós-AVC (OR2.9) }\end{array}$ \\
\hline $\begin{array}{l}\text { Paradiso, } \\
\text { Robinson, } \\
\text { 1998 }\end{array}$ & $\begin{array}{l}30 \mathrm{IH} \text {, admissões } \\
\text { consecutivas } \\
\text { e avaliadas }\end{array}$ & $\begin{array}{l}\text { Alteraçãode consciência } \\
\text { Dificuldademoderada ou } \\
\text { gravedecompreensão }\end{array}$ & $\begin{array}{l}\text { HAM-D } \\
\text { PSE } \\
\text { DSMIV }\end{array}$ & $\begin{array}{l}\text { Prospectivo 2semanas } \\
\text { apósoAVC }\end{array}$ & $\begin{array}{l}\text { Mulheres > Homens, Mulheres: diagnóstico psiquiátrico anteriore } \\
\text { prejuízo cognitivo ( + AVC esquerdo), Homens: mais jovens, prejuízo } \\
\text { atividades de vida diária e funcionamento social }\end{array}$ \\
\hline $\begin{array}{l}\text { Singhetal. } \\
2000^{19}\end{array}$ & $\begin{array}{l}449 R \\
81 \text { avaliados }\end{array}$ & $\begin{array}{l}\text { AVC bilateral,AVC prévio } \\
\text { Hemorragiasubaracnóide } \\
\text { AVCvertebrobasilar } \\
\text { Prejuízo cognitivo }\end{array}$ & $\begin{array}{l}M A D R S \geq 7 \\
Z U N G \geq 50\end{array}$ & $\begin{array}{l}\text { Prospectivo I,3,12 } \\
\text { meses apósoAVC }\end{array}$ & Grau de dependência: capacidade funcional nas atividades de vida diária \\
\hline
\end{tabular}

$\mathrm{A}=$ pacientes ambulatoriais, $\mathrm{C}=$ população de comunidade, $\mathrm{H}=$ pacientes hospitalares, $\mathrm{R}=$ pacientes de unidade de reabilitação. $\mathrm{BDI}=$ Inventário de depressão de $\mathrm{Beck}, \mathrm{CIDI}=\mathrm{Composite}$ International Diagnostic Interview, DSM = Manual diagnóstico e estatístico dos transtornos mentais, GHQ = General health questionnaire, HAMD = Escala para depressão de Hamilton, MADRS = Escala de avaliação para depressão de Montgomery-Asberg, $P A S=$ Psychiatric assessment schedule, PSE = Present state examination, $S C I D=$ Entrevista clinica estruturada para o DSM, ZUNG = Escala de auto-avaliação da depressão de Zung 
funcional foi responsável por $48 \%$ da variância na avaliação da depressão leve e moderada segundo o estudo de $\mathrm{Ng}$ et al. ${ }^{14}$. A cada unidade reduzida na escala de Barthel aumentava-se em $80 \%$ a probabilidade do paciente estar deprimido.

Em pacientes hospitalizados, no período agudo do pós-AVC, a contribuição do prejuízo funcional para o desenvolvimento da depressão mostrou ser fraco, sendo responsável por $10 \%$ da variância segundo o estudo de Robinson et al. ${ }^{9}$, enquanto no sexto mês a contribuição da associação aumentou ${ }^{17}$.

No entanto, em pacientes da comunidade, o prejuízo funcional detectado no primeiro mês do pós-AVC mostrou ser um preditor da depressão no terceiro mês ${ }^{18} \mathrm{e}$, também, foi o correlato mais forte para a sintomatologia depressiva tardia, entre o período de três a cinco anos após o AVC ${ }^{19}$. Agravidade do prejuízo funcional chegou a aumentar em 16 vezes a probabilidade do paciente ter depressão 20 .

\section{Prejuízo cognitivo}

O prejuízo cognitivo presente no primeiro mês pós-AVC também tem sido associado à depressão ${ }^{21}$. Nas duas primeiras semanas após - AVC encontrou-se uma associação entre o prejuízo cognitivo e a depressão $0^{9}$ e, ao longo de seis meses, houve um aumento desta associação ${ }^{17}$. O prejuízo cognitivo, entretanto, pode na realidade ser um sintoma depressivo. Um ensaio duplo cego realizado por Kimura et al..$^{22}$ mostrou que o déficit cognitivo melhorou após o tratamento da depressão.

\section{História de depressão}

A história pregressa de depressão tem sido apontada como um importante fator de risco para a depressão pós-AVC ${ }^{21}$. Pacientes com história de depressão apresentaram um risco 2,3 vezes maior de desenvolverem depressão pós-AVC equase trêsvezes mais de apresentarem depressão maior ${ }^{18}$. No estudo $\mathrm{Ng}$ et al. ${ }^{14}$, a história pregressa de depressão estava fortemente associada à depressão pós-AVC e foi o fator responsável por $64 \%$ da variância na avaliação do diagnóstico da depressãolevee moderada. Todos os episódios pregressos de depressão, apresentados pelos pacientes estudados, foram caracterizados como reativos pelos autores e haviam remitido, espontaneamente, sem tratamento. Estes pacientes tinham uma probabilidade de ter depressão no pós-AVC quase setevezes maior em relação aos pacientes sem história de depressão. O estudo transversal de Morris et al..$^{23}$ detectou que os pacientes com história de transtornos afetivos ou ansiosos tinham um risco quase quatro vezes maior de estarem deprimidos (OR 3.83) após o AVC, e para a depressão maior, o risco era quase seis vezes maior (OR 5,7).

\section{Idade}

A idade tem mostrado uma associação diferente com a depressão dependendo do período pós-AVC. Os pacientes mais jovens mostraram uma maior propensão para desenvolver depressão de forma mais grave durante o período agudo pós-AVC'. Por outro lado, no período tardio pós-AVC, nos pacientes internados em centros de reabilitação, a idade mais avançada mostrou ser um fator preditor independente (OR I.I) para a depressão maior ${ }^{23}$.

\section{Sexo}

Em alguns estudos a proporção entre homens e mulheres acometidos pela depressão pós-AVC difere da encontrada para a depressão na população geral. Nesta, a depressão acomete mais mulheres do que homens, na proporção de 2 para I.

Acompanhando pacientes com AVC, na comunidade, Burvill et al. ${ }^{15}$ diagnosticaram depressão em $23 \%$ dos homens e $24 \%$ das mulheres. A proporção entre depressão maior e menor foi de 2,5:I nos homens e I,4:I nas mulheres; além disso, no quarto e no décimo segundo mês pós-AVC a depressão maior foi mais freqüente nos homens. $\mathrm{Na}$ amostra estudada por Morris et al.$^{23}$, os pacientes do sexo masculino tinham um risco aumentado (25 vezes), em comparação às mulheres, para apresentar depressão menor.

Por outro lado, o sexo feminino foi considerado fator de risco em pacientes de comunidade $^{|4,2|}$, aumentando em até oito vezes a probabilidade de desenvolver depressãa ${ }^{20}$. No estudo de Paradiso e Robinson ${ }^{24}$, que avaliaram especificamente as diferenças entre os gêneros, a depressão maior no período agudo pós-AVC foi mais freqüente nas mulheres do que nos homens, sendo que nas mulheres a história de diagnóstico psiquiátrico anterior e o prejuízo cognitivo estavam associados ao grau de depressão, enquanto a idade mais jovem, o prejuízo nas atividades de vida diária e no funcionamento social estavam associados à severidade da depressão nos homens.
Um perfil de prevalência da depressão pós-AVC conforme os gêneros, diferente do esperado, pode ser um indício de uma fisiopatologia específica para pelo menos um subgrupo de pacientes com depressão pós-AVC, realçando a necessidade de estudos para esclarecer seu mecanismo.

\section{História de AVC prévio}

AVC prévio tem sido associado ao desenvolvimento da depressão pós-AVC tanto em populações de centro de reabilitação quanto da comunidade ${ }^{16,21}$.

\section{Hipercortisolemia}

A hipercortisolemia tem sido detectada como um fator de vulnerabilidade para 0 desenvolvimento da depressão maior pósAVC. A não-supressão do cortisol pela administração de dexametasona tem sido considerada por alguns como um potencial instrumento diagnóstico e de acompanhamento da recuperação da depressão maior pós-AVC $25,26,27$.

A hipercortisolemia não mostrou associação com a depressão no pós-AVC agudo no estudo de Olsson et al. ${ }^{28}$, no entanto, foi um fator preditivo para a ocorrência de depressão maior no primeiro e terceiro anos pós-AVC, (canonical loading 0.95) ${ }^{29}$.

\section{Aspectos sociais}

Morar sozinho e a existência de uma pobre rede social presente antes e/ou depois do AVC são apontados como fatores de risco para o desenvolvimento da depressão pós-AVC $9,10,15,17,21$.

\section{Correlato neuroanatômico}

As características neuroanatômicas do AVC constituem um fator importante e significativo para a ocorrência da depressão pósAVC. Muitas investigações têm destacado a localização do AVC como um fator de risco para o desenvolvimento da depressão mesmo quando avaliado em conjunto com os demais fatores de risco (Tabela 3). As características do AVC que têm sido avaliadas são: lateralidade, localização anterior/posterior, volume e regiões e estruturas anatômicas onde se instalou o AVC.

\section{Lateralidade}

O hemisfério onde se deu o AVC é uma das características que tem sido correlacionadas a uma maior freqüência e maior gravidade da depressão pós-AVC. Os estudos de 
Tabela 3 - Estudos de investigação de correlatos neuroanatômicos para a depressão pós-AVC

\begin{tabular}{|c|c|c|c|c|c|c|c|c|c|}
\hline \multirow[t]{2}{*}{ Estudo } & \multirow{2}{*}{ População } & \multirow{2}{*}{ Fatores de exclusão } & \multirow{2}{*}{ Características do estudo } & \multicolumn{6}{|c|}{ Correlato neuroanatômico } \\
\hline & & & & $A^{*}$ & $\mathbf{P}$ & V & HE & HD & Locais \\
\hline $\begin{array}{l}\text { Robinson } \\
\text { et al. } 1983^{30}\end{array}$ & $\begin{array}{l}\mathrm{I} 84 \mathrm{H} \\
\text { admissões } \\
\text { consecutivas } \\
\text { 48avaliados }\end{array}$ & $\begin{array}{l}\text { História psiquiátrica, alteração de } \\
\text { consciência, afasia, canhoto, abuso } \\
\text { deálcool edrogas, história familiar } \\
\text { psiquiátrica, lesão cerebral prévia } \\
\text { aoAVC }\end{array}$ & $\begin{array}{c}\text { Corte-transversal } \\
\text { TC } \\
\text { 2 semanas pós-AVC } \\
\text { PSE/DSMIII/ZUNG/HAMD }\end{array}$ & +++ & + & + & +++ & + & - \\
\hline $\begin{array}{l}\text { Robinson } \\
\text { et al. } 1984^{31}\end{array}$ & $\begin{array}{l}\text { I23Hadmissões } \\
\text { consecutivas } \\
\text { 36avaliadas }\end{array}$ & $\begin{array}{l}\text { Canhoto, lesões múltiplas do AVC, } \\
\text { história pessoal de tratamento } \\
\text { psiquiátrico ou diagnóstico atual, } \\
\text { injúriacerebral prévia, } \\
\text { AVC não visualizado na TC }\end{array}$ & $\begin{array}{c}\text { Corte-transversal } \\
\text { TC } \\
\text { 2semanaspós-AVC } \\
\text { PSE/DSMIII/ZUNG/HAMD }\end{array}$ & +++ & + & + & +++ & + & - \\
\hline $\begin{array}{l}\text { Robinson } \\
\text { et al. } 1984^{17}\end{array}$ & $\begin{array}{l}103 \mathrm{H} \\
6 \mathrm{I} \text { avaliados }\end{array}$ & Alteração de consciência eafasia & $\begin{array}{c}\text { Prospectivo } \\
\text { TC,2 2semanas,3 3e6mesespós-AVC } \\
\text { PSE/DSMIII/ZUNG/HAMD }\end{array}$ & + & - & - & + & - & - \\
\hline $\begin{array}{l}\text { Sinyor } \\
\text { etal. } 1986^{37}\end{array}$ & $\begin{array}{l}\text { I66R } \\
\text { admissões } \\
\text { consecutivas } \\
35 \text { avaliados }\end{array}$ & $\begin{array}{l}\text { Afasiae dificuldade de compreensão, } \\
\text { condiçõesmédicas potencialmente } \\
\text { complicadoras, AVCprévio, idade } \\
85 \text { anos, idiomaestrangeiro, história } \\
\text { psiquiátricaanterior, uso atual de } \\
\text { antidepressivos, nãovisualização do } \\
\text { AVC na TCouvisualização demais } \\
\text { deuminfarto. }\end{array}$ & $\begin{array}{c}\text { Corte-transversal } \\
\text { TC } \\
55 \text { dias pós-AVC } \\
\text { ZUNG/BDI/HCSL/CDI/NRS }\end{array}$ & + & + & + & - & - & - \\
\hline $\begin{array}{l}\text { Dam } \\
\text { etal. } 1989^{35}\end{array}$ & $\begin{array}{l}92 \mathrm{H} / \mathrm{A} \\
\text { avaliados }+ \\
30 \text { controles }\end{array}$ & $\begin{array}{l}\text { Afasiasevera } \\
\text { Doençapsiquiátrica } \\
\text { Doençagrave }\end{array}$ & $\begin{array}{c}\text { Caso-controle } \\
\text { TC } \\
35 \text { dias }(8-1280 \text { dias }) \\
\text { RDC/HAM-D/BDI,8AVCs bilaterais }\end{array}$ & + & - & - & - & + & - \\
\hline $\begin{array}{l}\text { House } \\
\text { etal. } 1990^{38}\end{array}$ & $\begin{array}{l}116 \mathrm{C} \\
73 \text { avaliados }\end{array}$ & $\begin{array}{l}\text { AVCbilateraleprévio } \\
\text { Doença psiquiátrica durante } \\
\text { oanoanterior ao AVC }\end{array}$ & $\begin{array}{c}\text { Prospectivo } \\
\text { TC } \\
\text { I,6,12 meses, PSE/DSMIII/BDI }\end{array}$ & + & - & - & + & + & - \\
\hline $\begin{array}{l}\text { Sharpe } \\
\text { et al. } 1990^{39} \\
1994^{20}\end{array}$ & $\begin{array}{l}515 \mathrm{C} \\
60 \text { avaliados }\end{array}$ & $\begin{array}{l}\text { Mais de um infarto visualizado na TC, } \\
\text { infarto bilateral, hemorragia } \\
\text { subaracnóidea.AVCprévio }\end{array}$ & $\begin{array}{c}\text { Transversal } \\
\text { TC } \\
\text { 3-5 anospós-AVC } \\
\text { PSE/SCID/DSMIIIR/HAD }\end{array}$ & - & - & + & - & - & - \\
\hline $\begin{array}{l}\text { MacHale } \\
\text { etal. } 1998^{36}\end{array}$ & $\begin{array}{l}\text { I87H/A, admissões } \\
\text { consecutivas } \\
\text { I } 45 \text { avaliados } \\
55 \text { com lesãoúnica } \\
\text { naTCavaliados }\end{array}$ & $\begin{array}{l}\text { Historiadedepressão, Lesões } \\
\text { múltiplas, Período pós-AVC > I mês } \\
\text { Déficitcognitivograve, afasiagrave }\end{array}$ & $\begin{array}{l}\text { Ensaio randomizado } \\
\text { Corte-transversal } \\
\text { TC,6meses pós-AVC } \\
\text { SADS/DSMIV/HAD }\end{array}$ & + & - & + & - & + & - \\
\hline $\begin{array}{l}\text { Beblo } \\
\text { etal. } 1999^{34}\end{array}$ & $\begin{array}{l}20 \mathrm{H} / \mathrm{R} \\
\text { pacientescom } \\
\text { depressãoavaliados }\end{array}$ & $\begin{array}{l}\text { AVC bilateral, Doençasevera } \\
\text { História de doença neurológicae } \\
\text { psiquiátrica, afasia, AVC > 4 meses } \\
\text { Álcooledrogas }\end{array}$ & $\begin{array}{c}\text { Corte-transversal } \\
\text { TC } \\
\text { 4mesespós-AVC } \\
\text { SCID/DSMIIIR/BDI/MADRS/CDS }\end{array}$ & - & - & - & + & + & $\begin{array}{l}\text { Gânglios } \\
\text { dabase }\end{array}$ \\
\hline $\begin{array}{l}\text { Vataja } \\
\text { etal.200140 }\end{array}$ & $\begin{array}{l}486 \mathrm{H} \\
275 \text { avaliados }\end{array}$ & $\begin{array}{l}\text { Afasia,surdez, doençagrave, obesidade, } \\
\text { claustrofobia, contra-indicaçãoparaRM }\end{array}$ & $\begin{array}{c}\text { Corte-transversal } \\
\text { RM } \\
\text { 3 mesespós-AVC } \\
\text { PSE/SCAN/DSMIIIR/CIDIO/MADRS }\end{array}$ & - & - & - & + & - & $\begin{array}{l}\text { Gânglios } \\
\text { dabase }\end{array}$ \\
\hline
\end{tabular}

$A=$ População ambulatorial, $H=$ População hospitalar, $R=$ População de unidade de reabilitação, $C=$ População de comunidade, $T C=$ Tomografia computadorizada, $R M=$ Ressonância magnética, $A *$ Anterior, $P=$ Posterior, $\mathrm{V}=$ Volume, $\mathrm{HE}=$ Hemisfério esquerdo, $\mathrm{HD}=$ Hemisfério direito $\mathrm{BDI}=$ Inventário de depressão de beck, $\mathrm{CDS}=$ Cornell depression scale, CIDI = Composite international diagnostic Interview, $\mathrm{DSM}=$ Manual diagnóstico e estatístico dos transtornos mentais, GHQ = General health questionnaire, HAD = Escala hospitalar para ansiedade e depressão, HAMD = Escala para depressão de Hamilton, MADRS = Escala de avaliação para depressão de montgomery-Asberg, NRS = Nurses'rating scale, $P A S=$ Psychiatric assessment schedule, PSE = Present state examination, RDC $=$ Research diagnostic criteria, $S A D S=$ Roteiro para disturbios afetivos e esquizofrenia, $S C A N=$ Schedules for clinical assessment in neuropsychiatry, SCID = Entrevista clínica estruturada para o DSM, ZUNG = Escala de auto-avaliação da depressão de Zung. 
Robinson et al. ${ }^{30,31}$ foram os primeiros a mostrar que pacientes com AVC no hemisfério esquerdo $(\mathrm{HE})$, quando comparados com aqueles com AVC no hemisfério direito (HD), apresentavam em maior freqüência depressão, de forma mais grave e, apresentando significativamente mais sintomatologia vegetativa, ou seja: despertar precoce, diminuição do apetite e perda de peso ou redução do desejo sexual. No estudo de Astrom et al..$^{32}$ o fator determinante mais importante para o desenvolvimento da depressão até o terceiro mês do pós-AVC foi a localização do AVC no HE. Quando se associou a anterioridade à lateralidade esquerda do AVC, a freqüência da depressão foi I 0 vezes maior nestes pacientes do que nos pacientes com AVC no HD. Outros estudos considerando estruturas anatômicas acometidas pelo AVC têm confirmado a associação entre o acometimento do $\mathrm{HE}$ e uma freqüência da depressão maior mais elevada, no período pós-AVC agudo ${ }^{33,34}$.

Acumula-se a estes achados outros que mostram uma associação mais tardia entre AVC no HD e a depressão $0^{35}$. De acordo com o estudo de MacHale et al..$^{36}$, no sexto mês pós-AVC, os pacientes com AVC no HD apresentavam um risco seis vezes maior de ficarem deprimidos equatro vezes maior de desenvolverem depressão maior, do que os pacientes com AVC no HE.

Alguns estudos não encontraram associação entre a lateralidade do AVC e a ocorrência de depressão em pacientes de centro de reabilitação $0^{37}$ e da comunidade ${ }^{38,39}$.

\section{Anterioridade}

Alocalização do AVC, estudada de acordo com sua instalação anterior ou posterior, nos hemisférios cerebrais, tem sido analisada em grande parte dos estudos conforme os critérios elaborados por Robinson et al..$^{30}$.

No período agudo, a proximidade do AVC ao pólo frontal, no HE, mostrou uma relação significativa com a depressão maior, na sua freqüência ${ }^{35}$ e na presença de sintomatologia mais grave, sendo responsável por $50 \%$ a $70 \%$ da variância no estudo de Robinson et al..$^{30,31}$ Essa relação se manteve ao longo dos seis meses posteriores ao $\mathrm{AVC}$ no $\mathrm{HE}$, enquanto no HD houve uma inversão da associação entre a gravidade da depressão e o AVC posterior para $\mathrm{o}$ anterior ${ }^{17}$. A anterioridade do AVC no HD também tem sido detectada como um fator associado à depressão $0^{35}$, acarretando um risco aumentado de 5,7 vezes para desenvolver a depressão maior ${ }^{36}$. No entanto, achados iniciais indicaram que a localização posterior do AVC no HD estava relacionada à depressão, principalmente àquela sem sintomatologia vegetativa, quando comparado $\mathrm{HD}$ e HE $\mathrm{HE}^{30,31,37}$.

Já estudos com pacientes de centros de reabilitação e da comunidade verificaram, respectivamente, que; aproximadamente dois meses após o AVC, os infartos anteriores de $\mathrm{HE}$ e anterior e posterior de HD, estavam relacionados à depressão $0^{37}$; e que havia uma associação, apesar de fraca, entre a depressão e a anterioridade do AVC em ambos hemisférios, quando todas as localizações foram estudadas de forma global no primeiro, sexto e décimo segundo mês pós-AVC ${ }^{38}$.

\section{Volume}

$O$ volume da região acometida é outra característica relacionada à ocorrência da depressão pós-AVC ${ }^{36}$. O volume do AVC associado à determinada localização mostra maior correlação com a depressão, como visto nos pacientes com depressão pós-AVC que tinham AVC localizado no hemisfério esquerdo em região anterior ${ }^{30,23}$.

Embora a associação entre o volume do AVC ea depressão permita formular a hipótese de que o maior prejuízo funcional gerado por estes AVCs explicaria a depressão, evidências têm indicado não ser este o principal motivo da associação. No estudo de Sharpe et al. ${ }^{39}$, o volume do AVC se correlacionou com os escores de sintomas depressivos ao longo dos trêsa cinco anos de seguimento, sugerindo uma possível associação da maior incapacidade causada por estes AVCs e a depressão. Entretanto, em estudo posterior, Sharpe et al. ${ }^{20}$ detectaram a permanência da associação entre o volume do AVC (OR:6.6) ea depressão tardia, após ajustes para prejuízo funcional e sexo. Este achado sugere que um mecanismo neural relacionado ao volume influi na manifestação depressiva independente do prejuízo funcional. Corrobora o achado anterior o estudo de MacHale et al. ${ }^{36}$, quenão encontrouassociaçãosignificativa entre prejuízo funcional e depressão.

Alguns estudos não confirmaram a correlação entre o volume do AVC e a severidade da depressão, indicando ainda existir a necessidade de estudos a respeito ${ }^{33,35}$.

\section{Localização neuroanatômica}

A investigação de áreas neuroanatômicas acometidas pelo AVC como fatores de risco para a depressão pós-AVC é um método que pode facilitar uma comparação dos resultados dos diferentes estudos e viabilizar um maior conhecimento sobre a fisiopatologia da depressão pós-AVC.

OAVClocalizado nosgânglios da base do HE esuas vizinhanças mostrou umaassociação significativa coma ocorrência de depressão maio ${ }^{33,34}$, enquanto infartos localizados na substância branca periventricular e parte posterior do pálido no $\mathrm{HD}$ foram associados à distimia ${ }^{34}$.

Ao comparar pacientes com e sem depressão pós-AVC, utilizando ressonância magnética de forma sistemática, o estudo de Vataja et al. ${ }^{40}$ verificou que os pacientes com depressão tinham commais freqüência infartos afetando o circuito prefrontosubcortical ou algumas de suas estruturas: caudado, pálido, joelho da cápsula interna e cápsula anterior, especialmente no HE. $\mathrm{O}$ AVCem região da amígdala, apesar de raro, foi considerado super-representativo, pois cinco dos únicos seis pacientes com infarto nesta região estavam deprimidos. Na análise multivariada os correlatos neuroanatômicos independentes da depressão pós-AVC foram o número de infartos no joelho da cápsula interna no HE (OR 3.2) e o número de infartos no pálido de qualquer hemisfério (OR I.6).

\section{Outros fatores associados à depressão}

Alguns fatores associados à depressão na idade avançada foram avaliados na população de pacientes com AVC, tais como: a atrofia cortical, subcortical elesões desubstânciabranca. Aatrofia cortical e subcortical não contribuíram para o desenvolvimento da depressãono pós-AVC agudo. No entanto, tais elementos detectados pela tomografia computadorizada feita no episódio índice e depois de três anos do AVC estavam associados à depressão maior que surge tardiamente, sendo observados tanto no grupo de pacientes deprimidos inicialmente eque continuavam com depressão no período tardio, assim como no grupo de pacientes que desenvolveu a depressão maior no período tardio ${ }^{32}$.

Aatrofiacerebral não se correlacionou com a gravidade da depressão de acordo com o estudo de Herrmann et al. ${ }^{33}$. No estudo de Vataja etal. ${ }^{40}$ nãoforam encontradas diferenças entreos grupos de pacientes comesem depressão quanto à atrofia cerebral e lesões de substância branca. 


\begin{tabular}{|c|c|c|c|c|c|}
\hline \multicolumn{6}{|c|}{ Tabela 4 - Impacto da depressão no período pós-AVC } \\
\hline Estudo & População & Fatores de exclusão & Instrumentos & Características & Impacto \\
\hline $\begin{array}{l}\text { Sinyior } \\
\text { et al. } 1986^{41}\end{array}$ & $\begin{array}{l}\text { I66admissões consecutivas } \\
64 \text { avaliados, centro de } \\
\text { reabilitação, primeiroAVC }\end{array}$ & $\begin{array}{l}>85 \text { anos condições médicas } \\
\text { afasia, historia psiquiátrica prévia } \\
\text { uso atual deantidepressivos }\end{array}$ & $\begin{array}{c}\text { ZUNG }>50 \\
\text { BHS } \\
\text { HSCL } \\
\text { CDI,NRS }\end{array}$ & $\begin{array}{l}\text { Prospectivo, período } \\
\text { pós-AVC: } 2 \text { meses, avaliações } \\
\text { naadmissão, altae } 6 \text { semanas } \\
\text { apósalta }\end{array}$ & $\begin{array}{l}\text { Maior prejuízo funcional naadmissão } \\
\text { e na alta. Após alta, queda do nível } \\
\text { funcional após } 6 \text { semanas. }\end{array}$ \\
\hline $\begin{array}{l}\text { Eastwood } \\
\text { et al. } 1989^{13}\end{array}$ & $\begin{array}{l}\text { 106admissões, } 87 \text { incluídos } \\
\text { centro dereabilitação }\end{array}$ & $\begin{array}{l}\text { Condiçãomédicainstável } \\
\text { Déficitcognitivograve }\end{array}$ & $\begin{array}{l}\text { SADS } \\
\text { ZUNG } \\
\text { GDS } \\
\text { HAM-D } \\
\text { RDC }\end{array}$ & $\begin{array}{l}\text { Prospectivo, caso-controle } \\
\text { avaliaçõesfeitas naadmissão } \\
4 \text { meses após a admissão } \\
\text { ena alta }\end{array}$ & $\begin{array}{l}\text { Maior tempo de permanência } \\
\text { hospitalar nafaseaguda }(p<0.05) \\
\text { Maior comprometimento } \\
\text { funcional }(p<0.01)\end{array}$ \\
\hline $\begin{array}{l}\text { Parikh } \\
\text { et al. } 1990^{42}\end{array}$ & $\begin{array}{l}\text { Admissõeshospitalares: } \\
\text { 63avaliados, Ambulatorial }\end{array}$ & $\begin{array}{l}\text { Outro AVC nos } 2 \text { anos de } \\
\text { seguimento condição médica } \\
\text { que afete atividades devida } \\
\text { diária, afasiagrave }\end{array}$ & $\begin{array}{l}\text { HAM-D } \\
\text { ZUNG } \\
\text { PSE } \\
\text { DSMIII }\end{array}$ & $\begin{array}{l}\text { Prospectivo } \\
\text { avaliações pós-AVC: } \\
2 \text { semanas, } 2 \text { anos }\end{array}$ & $\begin{array}{l}\text { Pior grau de capacidade das } \\
\text { atividadesfísicas e da linguagem } \\
2 \text { anos após oAVC }\end{array}$ \\
\hline $\begin{array}{l}\text { Burvill } \\
\text { et al. } 1995^{15}\end{array}$ & $\begin{array}{l}498 \\
248(4 \text { mês }) \\
233(12 \text { mês }) \\
\text { Comunidade }\end{array}$ & $\begin{array}{l}\text { Demênciasevera } \\
\text { afasiasevera }\end{array}$ & $\begin{array}{l}\text { PAS } \\
\text { DSMIII } \\
\text { PSE }\end{array}$ & $\begin{array}{l}\text { Prospectivo: I ano } \\
\text { avaliações, no } 4^{\circ} \text {. } \\
\text { e } 12^{\circ} \text {. mês }\end{array}$ & $\begin{array}{l}\text { Proporção demortalidade maior } \\
\text { entreos deprimidos (7.2\%)eos } \\
\text { ansiosos }(12.1 \%) \text { do quesem } \\
\text { diagnósticopsiquiátrico(3.4\%) }\end{array}$ \\
\hline King $1996^{43}$ & $\begin{array}{l}\text { 698altas de programas } \\
\text { de reabilitação } 86 \text { não } \\
\text { institucionalizadosavaliados }\end{array}$ & $\begin{array}{l}\text { Doençasneuromusculares } \\
\text { históriapsiquiátricaprévia }\end{array}$ & $\begin{array}{l}\text { CES-D } \\
\text { QOL } \\
\text { QLI }\end{array}$ & $\begin{array}{l}\text { Transversal, I a } 3 \text { anos pós-AVC } \\
\text { primeiro AVC ou outrosem } \\
\text { seqüelas/prejuízo }\end{array}$ & $\begin{array}{l}\text { 23\% referiam baixa satisfação } \\
\text { Correlação entre a depressão e baixa } \\
\text { qualidade devida global, e também } \\
\text { nos domínios psicológicose da } \\
\text { saúdeefuncional }\end{array}$ \\
\hline $\begin{array}{l}\text { Beekman } \\
\text { et al. } 1998^{6}\end{array}$ & $\begin{array}{l}3050 \text { (55-85 anos) } \\
\text { comunidade, I73 avaliados } \\
\text { para estudo das conseqüências }\end{array}$ & $\begin{array}{l}\geq 85 \text { anos, muito doente, } \\
\text { incapacidade cognitiva } \\
\text { queprejudiquea entrevista }\end{array}$ & CES-D & $\begin{array}{l}\text { Transversal,ocorrênciadeAVC } \\
\text { durante } 10 \text { anos de seguimento } \\
\text { deuma população }\end{array}$ & $\begin{array}{l}\text { Após controle defatores confundidores } \\
\text { impacto mais evidenteno bem-estar } \\
\text { (OR7.24), limitações nas atividades } \\
\text { diárias (OR2.79) e dias acamados } \\
\text { (OR3.28), depressão não esteve } \\
\text { associadaàmortalidadeprospectivamente }\end{array}$ \\
\hline $\begin{array}{l}\text { House } \\
\text { et al. 2001 }\end{array}$ & $\begin{array}{l}\text { I387admissões } \\
\text { hospitalares } \\
\text { consecutivas } \\
\text { 448avaliados }\end{array}$ & $\begin{array}{l}\text { Hemorragiasubaracnóide, } \\
\text { doenças concomitantes que } \\
\text { afetem os cuidados, prejuízo } \\
\text { cognitivo severo ou da } \\
\text { linguagem }\end{array}$ & $\begin{array}{l}\text { GHQ-28 } \\
\text { PSE } \\
\text { CID IO }\end{array}$ & $\begin{array}{l}\text { Prospectivo I,I2e } 24 \\
\text { mesesapósAVC }\end{array}$ & $\begin{array}{l}\text { Sintomas depressivos no primeiro mês } \\
\text { (OR2.4) associados à mortalidade no } \\
\text { primeiroe segundo ano pós-AVC, } \\
\text { comajuste paraidade, prejuízo } \\
\text { cognitivo, incontinênciaurinaria, } \\
\text { incapacidadefísica }\end{array}$ \\
\hline $\begin{array}{l}\text { Lewis etal. } \\
\left.200\right|^{45}\end{array}$ & $\begin{array}{l}\text { 643admissões hospitalares } \\
\text { eambulatoriais } \\
\text { 372avaliados }\end{array}$ & $\begin{array}{l}\text { Tumor cerebral, hemorragia } \\
\text { subaracnóide, doença crônica } \\
\text { debilitante, morte antes dos } 6 \\
\text { meses deavaliaçãa }\end{array}$ & $\begin{array}{c}\text { HAD } \\
\text { GHQ-30 } \\
\text { MASS }\end{array}$ & $\begin{array}{l}\text { Prospectivo } \\
\text { randomizado } \\
30 \text { diase } 6 \text { meses pós-AVC } \\
3 \text { a } 5 \text { anos de seguimento }\end{array}$ & $\begin{array}{l}\text { Redução da sobrevida( } 22 \% \text { faleceram } \\
\text { dentro de } 3 \text { anos) associadaa certas } \\
\text { atitudes sobrea doença: fatalismo } \\
\text { (ORI.07), desamparo/desesperança } \\
\text { (OR I.07) por cada ponto na escala } \\
\text { deMASS }\end{array}$ \\
\hline
\end{tabular}

$\mathrm{BHS}=$ Beckhopelessness scale, $\mathrm{CDI}=$ Composite depression index, $\mathrm{CES}-\mathrm{D}=$ Escala de rastreamento populacional para depressão, $\mathrm{DSM}=$ Manual diagnósticoe estatístico dos transtornos mentais, $\mathrm{GDS}=$ Escala de depressão em geriatria, $\mathrm{GHQ}=$ General health questionnaire, $\mathrm{HAD}=$ Escala hospitalar de ansiedade e depressão, $\mathrm{HAM}-\mathrm{D}=$ Escala para depressão de $\mathrm{Hamilton}, \mathrm{HSCL}=\mathrm{Hopkins}$ Symptom checklist, MASS = Mental adjustment to stroke scaLe, NRS = Nurses' rating scale, PSE = Present state examination, PAS = Psychiatric assessment schedule, QOL = Quality of Life, QLI = Quality of Life Index-strokeversion, $\mathrm{RDC}=$ Research diagnosticcriteria, $S A D S=$ Roteiro para disturbios afetivos e esquizofrenia, ZUNG = Escala de auto-avaliação da depressão de Zung

\section{Impacto da depressão}

A depressão pós-AVC traz significativo comprometimento para a evolução do paciente (Tabela 4). Embora em uma unidade de reabilitação os pacientes com depressão pósAVC possam apresentar recuperação semelhante àqueles não deprimidos; com a alta, e sem a assistência hospitalar intensiva, os deprimidos tendem a apresentar significativa queda da recuperação funcional em comparação com os não deprimidos ${ }^{41}$. Pacientes com depressão no período agudo pós-AVC podem ter as atividades da vida diária comprometidas por até dois anos, independente das variáveis demográficas, do acesso aos serviços de saúde, do tratamento físico especializado, das características do AVC, do funcionamento social e do prejuízo cognitivo ${ }^{42}$. Os pacientes deprimidos apresentaram um período de per- manência hospitalar mais longo na fase aguda e um maior grau de comprometimento funcional ao ingressar no centro de reabilitação ${ }^{13}$.

Em estudos de corte transversal, a depressão foi o fator mais fortemente associado à baixa qualidade de vida dos pacientes num período de um a três anos pós-AVC ${ }^{43}$. Também foi associada a um efeito negativo no domínio do bem-estar e à maior incapacitação mesmo após con- 
trole para grau de limitação funcional, declínio cognitivo, número de doenças concomitantes, idade, sexo e grau de escolaridade ${ }^{6}$.

Aocorrência da depressão após o AVC tem sido associada a um aumento de mortalidade ${ }^{15}$. Os sintomas de depressão no primeiro mês pós-AVC podem aumentar o risco de mortalidade em até duas vezes nos dois anos subseqüentes ${ }^{44}$. Apesar de fraca, há uma associação entreatitudes negativas dos pacientes comAVC e redução da sobrevida. O fatalismo, a desesperança e o desamparo foram associados a um maior risco de redução do tempo de vida pósAVC, independente de outros fatores de risco ${ }^{45}$.

\section{Terapêutica antidepressiva}

A clínica destes pacientes caracteriza-se pelo subdiagnóstico e subtratamento. Sabe-se que apenas $20 \%$ a $50 \%$ dos pacientes com depressão pós-AVC são diagnosticados por médicos não-psiquiatras ${ }^{46}$. $O$ subtratamento também pode ser um indício do subdiagnóstico. Ytterberg et al. ${ }^{47}$ constataram que grande parte dos pacientes deprimidos não foram diagnosticados e nem tratados, nos três meses posteriores ao AVC, mesmo recebendo assistência multidisciplinar especializada ou de um médico generalista. Em estudos diferentes, $17,4 \%$ e $38,7 \%$ dos pacientes haviam recebido tratamento específico para depressão ${ }^{9,15}$. $O$ não tratamento está associado às sérias conseqüências do impacto da depressão. Por outro lado, seu tratamento tem revelado um saldo positivo.

Vários estudos têm mostrado uma boa eficácia da terapêutica antidepressiva ${ }^{48}$. A nortriptilina mostrou ser superior ao placebo e à fluoxetina quanto à remissão da sintomatologia depressiva e tolerância, de acordo com estudo duplo cego randomizado realizado por Robinson et al. ${ }^{49} \mathrm{e}$, superior ao placebo de acordo com Kimura et al. ${ }^{22}$. Wiart et al. ${ }^{50}$ constataram uma resposta significativa dos pacientes à fluoxetina quando comparada ao placebo na redução da gravidade da depressão maior. O citalopram também tem mostrado ser efetivo e seguro no tratamento da depressão pós-AVC como detectado por Andersen et al. ${ }^{51,52}$. Estes estudos consideraram resposta ao tratamento quando os pacientes apresentavam uma redução de mais de $50 \%$ dos escores de gravidade e não mais preenchiam os critérios para depressão.
Alémdamelhoradadepressãocomaterapêuticaantidepressiva, verificou-sequea remissão da depressão, de acordo com o estudo de Chemerinski etal..$^{53}$, estavaassociadaà melhora do funcionamento medido pela atividade de vida diária (AVD). No caso do prejuízo cognitivo, considerado um dos fatores de risco paraa depressão pósAVC pode na realidade se tratar de uma conseqüência da depressão e melhorar com o tratamento antidepressivo como demonstrado no ensaio duplo cego realizado por Kimura etal. ${ }^{22}$.

\section{Depressão causando AVC}

Dois estudos prospectivos, um realizado noJapão e outro nos Estados Unidos, acompanharam um grande número de indivíduos moradores da comunidade durante 10 e seis anos, respectivamente. Ambos detectaram que a sintomatologia depressiva aumentava o risco chegando a ser considerado um fator preditor para a ocorrência do $\mathrm{AVC}^{54,55}$.

\section{Discussão}

Analisar a natureza da associação entre depressão e AVC é necessária para seu melhor entendimento e aprimorar a terapêutica. Para uma investigação é necessário levar em conta a variabilidade desta associação. $A$ depressão pode ser: I) uma reação psicológica ao AVC e suas limitações; 2) uma fase (primeira ou não) de um transtorno depressivo (recorrente ou não) desencadeado pelo AVC; 3) uma continuação de um estado depressivo que já se iniciara antes do AVC; 4) uma ocorrência ao acaso após o AVC, sem relação com o mesmo; 5) secundária à lesão do AVC emáreas e circuitos neurofisiológicos envolvidos na regulação do humor (depressão secundária)e, 6) a depressão pode ainda estar relacionada ao AVC como um fator facilitador da sua ocorrência (depressão causando AVC).

\section{Limitações metodológicas}

A não consideração desta complexa associação entre depressão e AVC, acima citada, pode levar a conclusões equivocadas. Podemos tomar como exemplo a associação da depressão pós-AVC com o antecedente de depressão. A história de depressão tem sido considerada um fator de risco para a depressão pós-AVC em estudos realizados em unidades de reabilitação e comunidade ${ }^{11,16,15}$. À luz dos estudos prospectivos mostrando um maior risco de AVC em pacientes com antecedente de depressão, o AVC agiria como um fator desencadeante de um novo episódio depressivo, de um transtorno que havia contribuído para a ocorrência do AVC. Uma segunda possibilidade seria a depressão não ser um antecedente, mas estar em curso e o AVC ser um evento na sua evolução, uma vez que não existiria um intervalo sem sintomatologia depressiva antes ou depois do AVC. Em decorrência desta relação complexa, a inclusão destes pacientes no estudo acarreta de antemão um fator confundidor ao se analisar a fisiopatologia, os aspectos epidemiológicos e os aspectos psicológicos específicos da depressão pós-AVC. Conseqüentemente pode-se gerar uma freqüência da depressão pós-AVC superestimada. Estaríamos, também, englobando na análise transtornos psiquiátricos diferentes (depressão secundária ao AVC e depressão recorrente) com características distintas e distorcendo os achados que seriam exclusivos da depressão pós-AVC. Os aspectos metodológicos, principalmente nos estudos que objetivam investigar os correlatos neuroanatômicos, podem ser responsabilizados pela dificuldade em alcançar uma concordância e em possibilitar uma análise global dos resultados. As duas únicas revisões sistemáticas são concordantes em verificar a impossibilidade atual de se obter um consenso, através dos estudos publicados, sobre a associação de determinada localização do AVC e a depressão ${ }^{56,57}$. As amostras em geral são pequenas, heterogêneas e oriundas de locais diferentes, o que caracteriza e até seleciona o tipo de população a ser estudada, como é o caso das populações hospitalares, ambulatoriais, da comunidade ou de centros de reabilitação. Alguns autores assinalam o limite de poder da amostra devido ao pequeno número de indivíduos nos estudos ${ }^{38,39}$. Os critérios de seleção são variados, diferindo de um estudo para outro, assim como os períodos de avaliação. Os diferentes períodos avaliados mostram relações distintas da depressão com o AVC. Adepressão menor é mais freqüente em centros de reabilitação e em um período pósAVC mais tardio, enquanto a depressão maior predomina no período mais agudo, chegando a um pico de prevalência de $34 \%$ no sexto mês pós-AVC ${ }^{9-11}$. Outro freqüente viés metodológico é a utilização de escalas (que apenas indicam a gravidade) ao invés de entrevistas para a realização do diagnóstico de depressão. 
Um modelo fisiopatológico para a depressão secundáriaaoAVC. Emborahaja divergênciasentreos resultados das pesquisas sobre os correlatos neuroanatômicos, há uma tendência em aceitar queosinfartoslocalizadosno pólofrontal esquerdo e nos gânglios da base do hemisfério esquerdo estão associados a uma maior incidência e maior gravidade de depressão no período agudo pósAVC $^{58,59}$. Estesachados, inclusive, foramutilizados por alguns autores para formular a hipótese neurofisiopatológicadequeadepleçãoassimétrica decatecolaminas causadapelalesãoseria omecanismo da depressão pós-AVC ${ }^{30}$. O estudo de Mayberg et al. ${ }^{60}$, utilizando PET para análise dos receptores5-HT corticais(serotonina), constatou diferença derespostabioquímica entreos pacientes com AVC no HD e no HE. Enquanto no HD observou-se uma maior taxa de ligação de espiperona no córtex ipsilateral, sugerindo uma regulação para cima (upregulation) dos receptores 5-HT compensando uma redução do neurotransmissor, no HE não ocorria o mesmo fenômeno, sugerindo que a falta deste mecanismo compensatório, a regulação para cima dos receptores, é o responsável pela manifestação da depressão. Além deste achado, os mais altos escores indicando agravidade da depressãoestavamassociados a taxas de ligação aos receptores 5-HT mais baixas no córtex temporal esquerdo. Outro modelo explicativo enfoca o AVC nos gânglios da base. Nesta condição, a lesão dos gânglios da baseesuas vizinhanças causariamuma disfunção no sistema de transmissão das vias das aminas biogênicas, ascendentes edescendentes, responsáveis pela regulação do humor ${ }^{28,33}$.

\section{Conclusóes}

É preciso cautela ao se observar a presença de alguns fatores considerados de risco para a depressão pós-AVC. $O$ prejuízo cognitivo por exemplo, pode ser mais uma manifestação da depressão já instalada; 0 antecedente de depressão pode ser um dos fatores que causou 0 $A V C$; e a depressão subseqüente pode ser apenas a continuação de uma depressão prévia. O subdiagnóstico e conseqüente falta de tratamento agravam por mais tempo as repercussões negativas da depressão sobre os pacientes e seus familiares. Como as taxas de prevalência da depressão são altas não é possível menosprezar este fato na clínica. Os estudos mostram sérias dificuldades metodo- lógicas, mas indicam a existência de mecanismos neurofisiológicos na base da manifestação da depressão maior pósAVC. Evidências indicam que as lesões frontais esquerdas e dos gânglios da base possuem papel relevante na fisiopatologia da depressão pós-AVC. Os relatos de que o correlato neuroanatômico é o fator de risco mais importante para a depressão maior, principalmente no período agudo, ainda carecem de uma melhor definição. As limitações metodológicas mencionadas pelos diversos autores e presentes nos estudos podem servir de diretrizes para pesquisas futuras, refinando a investigação.

\section{Perspectivas}

Em função do estado atual de conhecimentos, pesquisas e programas devem ser realizados a fim de atingir os seguintes objetivos:

- Identificação de correlatos neuroanatômicos associados com a depressão maior em pacientes sem antecedente de depressão

- Investigação de variantes clínicas de depressão em função da localização do AVC

- Investigação de padrões de resposta à terapêutica antidepressiva em função da localização do AVC

- Investigação de associação entre refratariedade e localização do AVC

- Realização de programas para aumentar a freqüência do diagnóstico e tratamento da depressão pós-AVC pelo médico não-psiquiatra.

\section{SUMMARY}

Poststroke Depression: RISK factors AND ANTIDEPRESSANT TREATMENT

Depression is the most frequent psychiatric complication among stroke survivors. Several aspects have been indicated as risk factors for its occurrence. This review investigates the risk factors and the state of the art of the treatment for poststroke depression, in order to stimulate its detection and adequate treatment by the physician.

The point prevalence of Major Depression after stroke varies from $10 \%$ to $34 \%$, varying according to differences among the research methods. The length of poststroke period, characteristics of the sample, type of treatment received by patients and diagnostic criteria used can influence the reported prevalence of poststroke depression.

The risk factors that have been associated with the occurrence of poststroke depression, are: functional and cognitive impairment, previous history of depression and stroke, sex, age, hypercortisolism, poor social support and stroke neuroanatomic correlates. This one has supported the formulation of a pathophysiological mechanism for poststroke depression related with prefrontosubcortical circuits and neurotransmission of biogenic amines.

The depression has a harmful impact on stroke prognosis. It can cause a more severe functional impairment, retardation of the rehabilitation process, outcome complications, and a higher mortality risk. In addition, poststroke depression has not been accurately diagnosed and treated.

With the advantage of the magnetic ressonance, researchers should focus investigations on the association of specific cerebral regions with the depressive manifestation and treatment response. Methodological issues such as previous history of depression and the type of the depressive manifestation should be considered for analysis. [Rev Assoc Med Bras 2003; 49(4): 450-9]

KEY wORDS: Depression. Stroke. Risk Factors. Treatment.

\section{REFERÊNCIAS}

I. Fosltein FM, Maibeger R, McHugh PR. Mood disorder as a specific complication of stroke. J NeurolNerosurgery Psychiat 1977; 40: I0I8-20.

2. Robinson RG, Szetela B. Mood changes following left hemispheric brain injury. Ann Neurol 1981; 9:447-53.

3. Gainotti G, Azzoni A, Marra C. Frequency, phenomenology and anatomical - clinical correlates of major post-stroke depression. $\mathrm{Br}$ J Psychiatry 1999; I 75: I63-7.

4. Conselho Regional Medicina. Pro-Aim revela números da mortalidade.J Cremesp2002, I75:6.

5. Robinson RG. The clinical neuropsychiatry stroke: cognitive, behavioral, and emotional disorders following vascular brain injury. Cambridge: Cambridge University Press, 1999.

6. BeekmanATF,PenninxBWJH, DeegDJH, Ormel J,SmitJH, BraamAW, Van TilburgW. Depression in survivors of stroke: a community-based study of prevalence, risk factor and consequences. Soc Psychiatry Psychiatr Epidemiol I 998; 33:463-70.

7. Kessler RC, McGonagle KA, Zhao S, Nelson CB, Hughes M, Eshleman S, et al. . Lifetime and I 2-month prevalence of DSM-III-R psychiatric disorders in the United States. Arch Gen Psychiatry 1994; 51:8-19.

8. Balzer DG, Kessler RC, McGonagle KA, Swartz MS. The prevalence and distribution 
of major depression in a national community sample: the National Comorbidity Survey. Am J Psychiatry 1994; I 5 1:979-86.

9. Robinson RG, Starr LB, Kubos KL, Price TR. A two-year longitudinal study of post-stroke mood disorders: findings during the initial evaluation. Stroke 1983; |4:736-4 I.

10. Robinson RG, Starr BL, Price TR. A two-year longitudinal study of mood disorders following stroke. Prevalence and duration at six months follow-up. BrJ Psychiatry 1984; I44:256-62.

I I. Robinson RG, Bolduc PL, Price TR. Two-year longitudinal study of poststroke mood disorders: diagnosis and outcome at one and two years. Stroke 1987; 18:837-43.

12. Kotila M, Numminen H, Waltimo O, Kaste M. Depression after stroke. Results of the FINNSTROKE study. Stroke 1998; 29:368-72.

13. Eastwood MR, Rifat SI, Nobbs H, Ruderman ]. Mood disorder following cerebrovascular accident. Br J Psychiatry 1989; I 54: I 95-200.

14. Ng KC, Chan KL, Straughan PT. A study of post-stroke depression in a rehabilitative center. Acta Psychiatr Scand 1 995; 92:75-9.

15. Burvill PW, Johnson GA, Jamrozik KD, Anderson CS, Sewart-Wynne EG, Chakera TMH. Prevalence of depression after stroke: the Perth community stroke study. $\mathrm{Br}$ J Psychiatry 1995; 166:320-7.

16. Morris PLP, Shields RB, Hopwood MJ, Robinson RG, Raphael B. Are there two depressive syndromes after stroke? J Nerv Ment Dis 1994; 182:230-34.

17. Robinson RG, Starr LB, Lipsey JR, Rao K, Price T. A two-year longitudinal study of poststroke mood disorders: dynamic changes in associated variables over the first six months of follow-up. Stroke 1984; I 5:5 I 0-7.

18. Pohjasvaara T, Leppävuori A, Siira I, Vataja R, Kaste M, Erkinjuntti T. Frequency and clinical determinants of poststroke depression. Stroke 1998; 29:2311-17.

19. Singh A, Black SE, Herrmann N, Leibovitch FS, Ebert PL, Lawrence J, et al. Functional and neuroanatomic correlations in poststroke depression. The Sunnybrook Stroke Study. Stroke 2000; 31 :637-44.

20. Sharpe M, Hawton K, Seagroatt V, Bamford], House A, Molyneux A, Sandercock P, Warlow C. Depressive disorders in long term survivors of stroke. Associations with demographic and social factors, functional status and brain lesion volume. BrJ Psychiatry 1994; 164:380-6.

2I. Andersen G, Vestergaard K, Ingemann-Nielsen M, Lauritzen L. Risk factor for post-stroke depression. Acta Psychiatr Scand 1995; 92: 193-8.

22. Kimura M, Robinson RG, Kolier JT. Tratamento do comprometimento cognitivo depois de depressão pós-AVC. Um ensaio com tratamento duplo-cego. Stroke 2000; 31: | 482-6.

23. Morris PLP, Robinson RG, Raphael B, Samuels J, Molloy $\mathrm{P}$. The relationship between risk factors for affective disorder and poststroke depression in hospitalized stroke patients. Aust N Z J Psychiatry 1992; 26:208-I 7.

24. Paradiso S, Robinson RG. Gender differences in poststroke depression. J Neuropsychiatry Clin Neurosci 1998, 10:4 I-7.

25. Finklestein S, Benowitz LI, Baldessarini RJ, Arana GW, Levine D, Woo E, Bear D, Moya K, Stoll AL. Mood, vegetative disturbance, and dexamethasone suppression test after stroke. Ann Neurol I 982, 12:463-68.
26. LipseyJR, Robinson RG, Pearlson GD, Rao K, Price TR. The dexamethasone suppression test and mood following stroke. Am J Psychiatry 1985; 142:318-23.

27. Harney JH, Fulton C, Ross ED, Rush AJ. Dexamethasone suppression test and onset of poststroke depression in patients with ischemic infarction.J Clin Psychiatry 1993; 54:343-48.

28. Olsson T, Astrom M, Eriksson S, Forssell A. Hypercortisolism revealed by dexamethasone suppression test in patients (corrected) with acute ischemic stroke. Stroke 1989; 20: I 685-90.

29. Astrom M, Olsson T, Asplund K. Different linkage of depression to hypercortisolism early versus late after stroke. A 3-year longitudinal study. Stroke 1993; 24:52-7.

30. Robinson RG, Kubos KL, Starr LB, RaoK, PriceTR. Mood changes in stroke patients: relationship to lesion location. Comp Psychiatry 1 983; 24:555-66.

31. Robinson RG, Kubos KL, Starr LB, RaoK, Price TR. Mood disorders in stroke patients. Importance of location of lesion. Brain 1984; 107:81-93.

32. Astrom M, Adolfsson R, Asplund K. Major depression in stroke patients. A 3-year longitudinal study. Stroke 1993; 24:976-82

33. Herrmann M, Bartels C, Schumacher M, Wallesch CW. Poststroke depression. Is there a pathoanatomic correlate for depression in the postacute stage of stroke? Stroke 1995:26:850-6.

34. Beblo T, Wallesch CW, Herrmann M. The crucial role of frontostriatal circuits for depressive disorders in the postacute stage after stroke. Neuropsychiatry, Neuropsychol Behav Neurol 1999; I 2:236-46.

35. Dam H, Pedersen HE, Ahlgren P. Depression among patients with stroke. Acta Psychiatr Scand 1989; 80: I 18-24.

36. MacHale SM, O'Rourke SJ, Wardlaw JM, Dennis MS. Depression and its relation to lesion location after stroke. J Nerol Neurosurg Psychiatry 1998; 64:37|-4.

37. Sinyor K, Jacques P, Kaloupek DG, Becker R, Goldenberg M, Coopersmith H. Poststroke depression and lesion location. An attempted replication. Brain 1986; 109:537-46.

38. House A, Dennis M, Warlow C, Hawton K, Molyneux A. Mood disorders after stroke and their relation to lesion location. A CT scans study. Brain 1990; I I3: I I I 3-29.

39. Sharpe M, Hawton K, House A, Molyneux A, Sandercock P, Bamford J, Warlow C. Mood disorders in long-term survivors of stroke: associations with brain lesion location and volume. Psychol Med 1990; 20:8I 5-28.

40. Vataja R, Pohjasvaara T, Leppavuori A, Mantyla $\mathrm{R}$, Aronen HJ, Salonen O, Kaste M, Erkinjuntti $T$. Magnetic resonance imaging correlates of depression after ischemic stroke. Arch Gen Psychiatry 200I; 58:925-31.

4I. Sinyor D, Amato P, Kaloupek DG, Becker R, Goldenberg M, Coopersmith H. Post-stroke depression: relationships to functional impairment, coping strategies and rehabilitation outcome. Stroke 1986; 1 7: I 102-07.

42. Parikh RM, Robinson RG, Lipsey JR, Starkstein SE, Fedoroff JP, Price TR. The impact of poststroke depression on recovery in activities of daily living over a 2-year follow up. Arch Neurol 1990; 47:785-89.

43. King RB. Quality of life after stroke. Stroke 1996; 27: |467-72

44. House A, Knapp P, Bamford J, Vail A. Mortality at 12 and 24 months after stroke may be associated with depressive symptoms at month. Stroke 200 I; 32:696-70I .

45. Lewis SC, Dennis MS, O'Rourke SJ, Sharpe M. Negative attitudes among short-term stroke survivors predicts worse long-term survival. Stroke 2000:32: I 640-45

46. Schubert DSP, Burns R, Paras W, Sioson E. Increase of medical hospital length of stay buy depression in stroke and amputation patients: a pilotstudy. PsychotherPsychosom 1 992;57:6I-6

47. Ytterberg C, Malm SA, Britton M. How do stroke patients fare when discharged straight to their homes? A controlled study on the significance of hospital follow-up after one month. Scand J Rehab Med 2000; 32:93-6.

48. Starkstein SE, Lischinsky A. Diagnosis, phenomenology and treatment of poststroke depression. Rev Bras Psiquiatr 2002; 24:44-9.

49. Robinson RG, Schultz SZ, Castillo C, Kopel T, Kosier JT, Newman RM, Curdue K, Peetracca G, Starkstein SE. Nortriptyline versus fluoxetine in the treatment of depression and in short-term recovery after stroke: a placebocontrolled, double-blind study. Am JPsychiatry 2000; I57:35I-59.

50. Wiart L, Petiti H, Joseph PA, Mazaux JM, Barat M. Fluoxetine in early poststroke depression. A double-blind placebo-controlled study. Stroke 2000; $31: 1829-32$

5I. Andersen G, Vestergaard K, Lauritzen L. Effective treatment of poststroke depression with the selective serotonin reuptake inhibitor citalopram. Stroke 1994; 25: I 099- I I 04.

52. Andersen G, Vestergaard K, Riis JO Citalopram for post-stroke pathological crying. Lancet 1993; 342:837-39.

53. Chemerinski E, Robinson RG, ArndtS, Kosier JT. The effect of remission of poststroke depression on activities of daily living in a double blind randomized treatment study. J Nerv Ment Dis 200I; I89:42I-25.

54. Ohira T, Iso H, Satoh S, Sankai T, Tanigawa T, Ogawa $Y$, et al. Prospective study of depressive symptoms and risk of stroke among japanese. Stroke 200I; 32:903-8

55. Ostir GV, Markides KS, Peek MK, Goodwin JS. The association between emotional wellbeing and the incidence of stroke in older adults. Psychosom Med 200 I; 63:2 I 0-I 5

56. SinghA, Herrmann N, BlackSE. The importance of lesion location in poststroke depression: a critical review. Can J Psychiatry 1998:43:921-7.

57. Carson AJ, MacHale S, Allen K, Lawrie SM, Dennis M, House A, Sharpe M. Depression after stroke and lesion location: a systematic review. Lancet 2000; 356: I22-6.

58. Starkstein SE, Robinson RG. Aspectos neuropsiquiátricos das doenças vasculares cerebrais. In: Stuart C Yudofsky, Hales RE. Compêndio de neuropsiquiatria. Porto Alegre: Artes Médicas, 1996. p.264-74

59. Fricchione G, WeilburgJB, Murray GB. Neurology and neurosurgery. In: James R, Rundell, Wise MG.. Textbooks of consultation-liaison psychiatry. Washington: APP, 1996. p.696-7I9.

60. Mayberg HS, Robinson RG, Wong DF, Parikh $R$, Bolduc $P$, Starkstein SE, et al. . PET imaging of cortical S2 serotonin receptors after stroke: lateralized changes and relationship to depression. Am J Psychiatry 1988; 145:937-43.

Artigo recebido: 28/05/03

Aceito para publicação: 05/08/03 